\title{
Clinical Utility of Liquid Biopsy to Identify Genomic Heterogeneity and Secondary Cancer Diagnoses: A Case Report
}

\author{
Gregory Hemenway $^{a}$ Marni B. Tierno ${ }^{b}$ Reza Nejatic Romina Sosad \\ Matthew Zibelman ${ }^{d}$ \\ aDepartment of Medicine, Temple University, Philadelphia, PA, USA; ${ }^{b}$ Foundation \\ Medicine, Inc., Cambridge, MA, USA; ' Department of Pathology, Fox Chase Cancer Center, \\ Philadelphia, PA, USA; dDepartment of Hematology and Oncology, Fox Chase Cancer \\ Center, Philadelphia, PA, USA
}

\section{Keywords}

Circulating tumor DNA · Liquid biopsy · Comprehensive genomic profiling · CHEK2

\begin{abstract}
Liquid biopsy is a valuable tool in advanced and metastatic cancers for detection of genomic alterations in tumors that facilitate personalized targeted therapy approaches. Analyzing circulating tumor DNA (ctDNA) using next-generation sequencing (NGS) provides an opportunity to detect tumor genomic changes during therapy and capture inter- and intra-heterogeneity of genomically divergent cancer cell evolution. Herein, we present a patient with metastatic castration-resistant prostate cancer, with progression to soft tissues, bone, and regional lymph nodes, who was treated with abiraterone plus prednisone, with excellent prostatespecific antigen response. At the time of progression, NGS analysis of ctDNA using the FoundationOne ${ }^{\circledR}$ Liquid test revealed a CHEK2 mutation and a BRAF V600E mutation, the latter being exceedingly rare in prostate cancer. At the time of biochemical recurrence, the patient was referred to hematology for evaluation of chronic but stable thrombocytopenia prior to initiating new systemic therapy. Results of a bone marrow biopsy were consistent with hairy-cell leukemia, where the BRAF V600E mutation is considered the disease-defining mutation detectable in nearly all cases at diagnosis. In this case, liquid biopsy served as a noninvasive, highly sensitive approach to help reveal tumor genomic heterogeneity but also identified an unexpected genomic alteration leading to secondary cancer diagnosis and changes to treatment-related decision-making.
\end{abstract}


Hemenway et al.: Utility of Liquid Biopsy: A Case Report

\section{Background}

Comprehensive genomic profiling (CGP) using next-generation sequencing (NGS) has become routine in guiding treatment decisions for men with metastatic castration-resistant prostate cancer (mCRPC) following the recent approvals of novel targeted therapies such as poly (ADP-ribose) polymerase inhibitors (PARPi). Patients with mCRPC whose tumors harbor the germline or somatic homologous recombination repair (HRR) gene (i.e., BRCA1, BRCA2, and $A T M$ ) alterations are eligible to receive PARPi therapy due to improvements in outcomes and survival as noted in the PROfound and TRITON2 clinical trials [1-3]. CGP testing can identify genomic alterations within $B R C A 1$ and $B R C A 2$ genes included in the approvals for both olaparib and rucaparib, as well as 12 additional HRR genes included in the olaparib label $[4,5]$.

Genomic alterations that act as oncogenic drivers can be identified by CGP using a tumor tissue specimen or liquid biopsy. Analysis of tumor tissue remains the most sensitive method for capturing targetable genomic alterations [6, 7]; however, continuing advances in liquidbiopsy techniques have significantly improved the ability to detect multiple genomic alterations within circulating tumor DNA (ctDNA) following a simple blood draw [7, 8]. A possible advantage of using liquid biopsy over tumor tissue sampling is the ability to help reveal intratumor heterogeneity (the presence of more than one clone of cancer cells within a given tumor) and inter-tumor heterogeneity (the presence of different genomic alterations in different metastatic tumors from a single patient) $[7,9,10]$. However, inherent in this approach is a lack of specificity toward identifying the source of an individual mutation.

This case report illustrates the utilization of liquid biopsy in a patient with progressive mCRPC to capture genomic alterations from prostatic ctDNA, leading to the unexpected identification of a second primary malignancy of hairy-cell leukemia (HCL). This finding had significant implications in the treatment decisions for this patient and underscores both the power and limitations of this technology.

\section{Case Presentation}

A 63-year-old male was initially diagnosed in 2005 with clinical stage IIA (T1c, N0, M0) prostate adenocarcinoma at the age of 48 years, following a screening prostate-specific antigen (PSA) of $10.3 \mathrm{ng} / \mathrm{mL}$. Transurethral resection of the prostate showed left-sided Gleason 7 disease. A nuclear medicine bone scan did not reveal metastatic disease. He underwent a radical retropubic prostatectomy and pelvic lymph node dissection, where pathology revealed stage III disease (pT2c, N0, M0) with Gleason score $3+4=7$ with negative margins, lacking perineural invasion and focal invasion into the capsule. Past medical history was significant for prostate cancer in both his father and paternal grandfather. His paternal grandmother was diagnosed with leukemia. His mother had a history of breast cancer, and there is a history of ovarian cancer in one cousin.

In August 2015, he presented with biochemical recurrence with a measurable PSA of 0.58 $\mathrm{ng} / \mathrm{mL}$. Systemic imaging with a computerized tomography scan of the abdomen and pelvis and a nuclear medicine bone scan did not reveal any metastatic disease. He was treated with salvage, intensity-modulated radiation (6,800 cGy) to the prostate bed, which he completed concurrently with 4 months of androgen deprivation therapy (ADT). Postradiation PSA was undetectable.

One year after salvage radiation, the patient again developed biochemical recurrence with a PSA of $0.60 \mathrm{ng} / \mathrm{mL}$ (testosterone $282 \mathrm{ng} / \mathrm{dL}$ ). Repeat systemic imaging revealed an irregular soft tissue mass at the right suture line from the prior prostatectomy, concerning for metastatic disease. ADT with leuprolide was reinitiated. A prostate-specific membrane

\section{Karger'}


Table 1. Genomic alterations identified using FoundationOne ${ }^{\circledR}$ Liquid testing

\begin{tabular}{llllll}
\hline Gene & $\begin{array}{l}\text { Genomic } \\
\text { alteration }\end{array}$ & $\begin{array}{l}\text { Variant allele } \\
\text { frequency, \% }\end{array}$ & Pathway & $\begin{array}{l}\text { Mutation frequency } \\
\text { in prostate cases, } \%\end{array}$ & $\begin{array}{l}\text { Mutation frequency } \\
\text { in HCL cases, \% }\end{array}$ \\
\hline BRAF & V600E & 0.55 & MAPK & $2.2 ;$ V600E $<0.1$ & V600E $>97$ \\
NF1 & L1507fs*23 & 0.25 & RAS & 1.0 & 0 \\
CHEK2 & T367fs*15 & 50.85 & DNA damage repair & 1.8 & 2.2 \\
JAK2 & V617F & 0.37 & JAK-STAT & 0.3 & 0 \\
RB1 & R552fs*54 & 0.61 & $\begin{array}{l}\text { Cell cycle tumor } \\
\text { suppressor }\end{array}$ & 9.7 & 0 \\
\hline
\end{tabular}

Liquid biopsy results revealed the patient's specific genomic alterations including VAF. Relevant cell signaling pathways for each gene are provided as well as expected mutation frequency for each gene in prostate cases compared to HCL cases from published data.

VAF, variant allele frequency.

antigen positron emission tomography scan showed intense radiotracer uptake in a right internal iliac lymph node, as well as a nonpathologically enlarged but intensely radiotraceravid right external iliac lymph node, compatible with metastatic prostate disease (PSMARADS-5 and PSMA-RADS-4, respectively).

The PSA became undetectable with ADT but began rising after about 8 months despite persistent castrate state. New computerized tomography imaging revealed an interval decrease in size of the right pelvic sidewall soft-tissue mass with new sclerotic osseous lesions. A nuclear medicine bone scan confirmed multifocal active lesions consistent with bony metastasis. Daily abiraterone $1,000 \mathrm{mg}$ plus prednisone $5 \mathrm{mg}$ were added to his regimen and FoundationOne ${ }^{\circledR}$ Liquid testing was performed. Genomic findings included an unexpected BRAF V600E mutation, as well as mutations in NF1, CHEK2, JAK2, and RB1, which have been previously detected in genomic analyses of prostate cancer (Table 1) [6]. He tolerated abiraterone therapy with an excellent PSA response until October 2020 when his PSA rose again. Repeat imaging at that time revealed an interval increase in size of the metastatic right external iliac lymph node without active osseous disease. A timeline of this case presentation is outlined in Figure 1.

Discussions were initiated for future systemic therapy including a PARPi, given the CHEK2 mutation identified by liquid-biopsy testing. However, prior to changing systemic therapy, the patient was referred to hematology for evaluation of chronic but stable thrombocytopenia (Fig. 2).

A bone marrow biopsy was performed in October 2020 which was consistent with HCL (40-50\% of marrow cellularity). Immunohistochemistry staining of the bone marrow core sample revealed a large population of CD20+, PAX5+ (weak, subset) B-lymphocytes (Fig. 3). The BRAF V600E and CHEK2 mutations identified by FoundationOne ${ }^{\circledR}$ Liquid testing were consistent with this new diagnosis. His case was reviewed at a multidisciplinary tumor board. Given the indolent nature of the HCL and lack of cytopenias, the decision was made to monitor the HCL without active treatment, prioritizing treatment for his progressive prostate cancer. Subsequently, he continued on abiraterone/prednisone and was referred back to radiation oncology to receive stereotactic body radiation therapy to his right pelvic node 5,000 cGy in 5 fractions) with successful reduction in his PSA.

Due to this patient having metastatic prostate cancer and his significant family history of cancer, the CHEK2 mutation was further evaluated in November 2020 with germline genetic testing using CancerNext-Expanded testing. This showed germline heterozygosity for both pathogenic c.1100delC mutation and a likely pathogenic c.592+3A>T mutation.

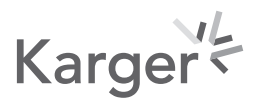




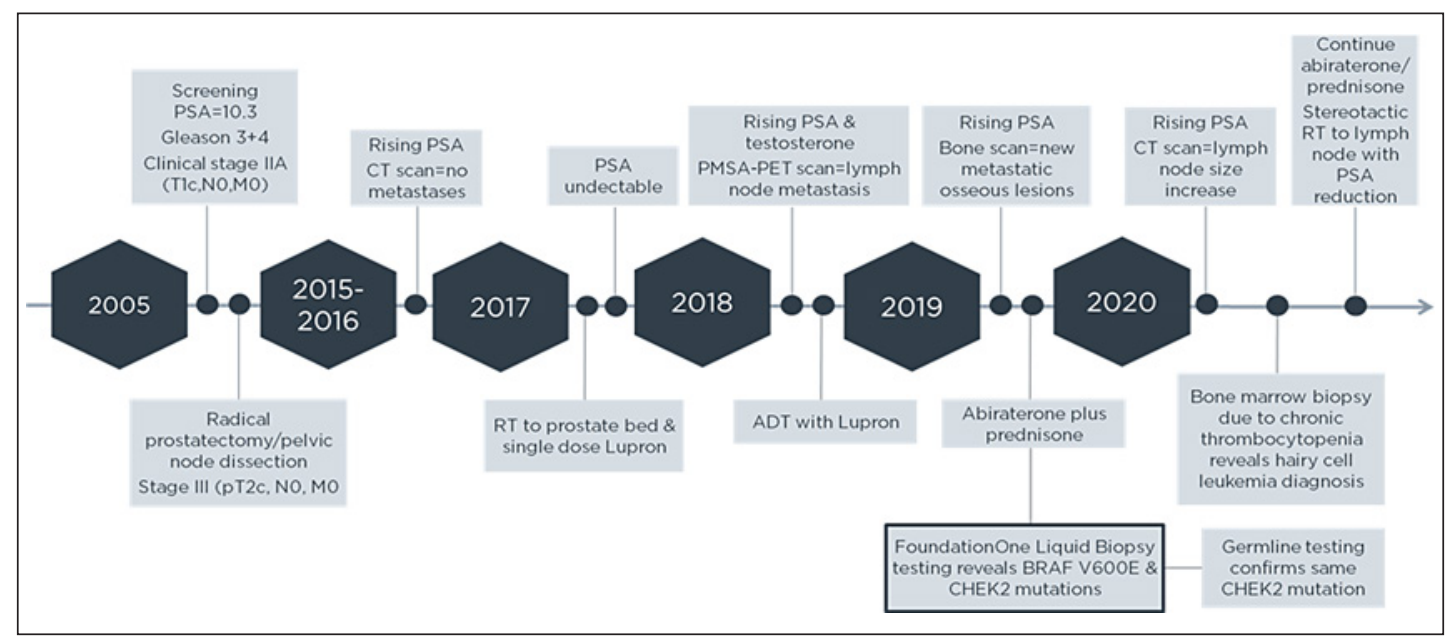

Fig. 1. Patient's clinical course from time of diagnosis of prostate cancer through incidental identification of second primary HCL. The patient's clinical presentation over time revealing timepoints of biochemical and radiographic progression, treatment regimens, and liquid-biopsy testing.

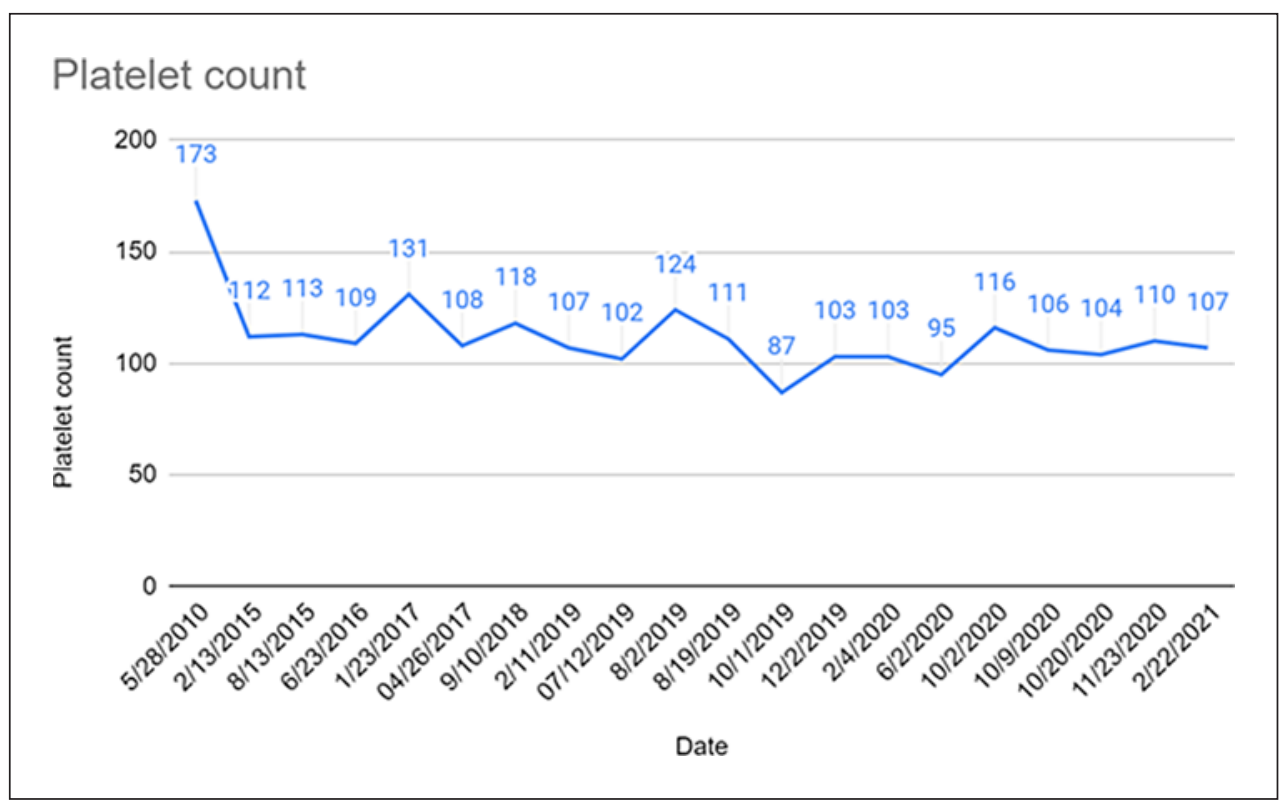

Fig. 2. Platelet count results obtained during patient's care. The patient's platelet count trend suggesting a slowly progressive bone marrow process resulting in mild thrombocytopenia.

\section{Discussion}

Biomarker-directed therapies now represent practical treatment options for mCRPC patients. Tumors that harbor HRR gene alterations can be targeted with PARPi therapy, while tumors with microsatellite instability-high or tumor mutational burden-high (TMB-H) status may be responsive to immune checkpoint inhibitors [1, 3-5, 11, 12]. This has led to increased utilization of tumor genomic profiling to identify appropriate biomarkers. Though the approval of rucaparib is limited to tumors with BRCA1 or BRCA2 

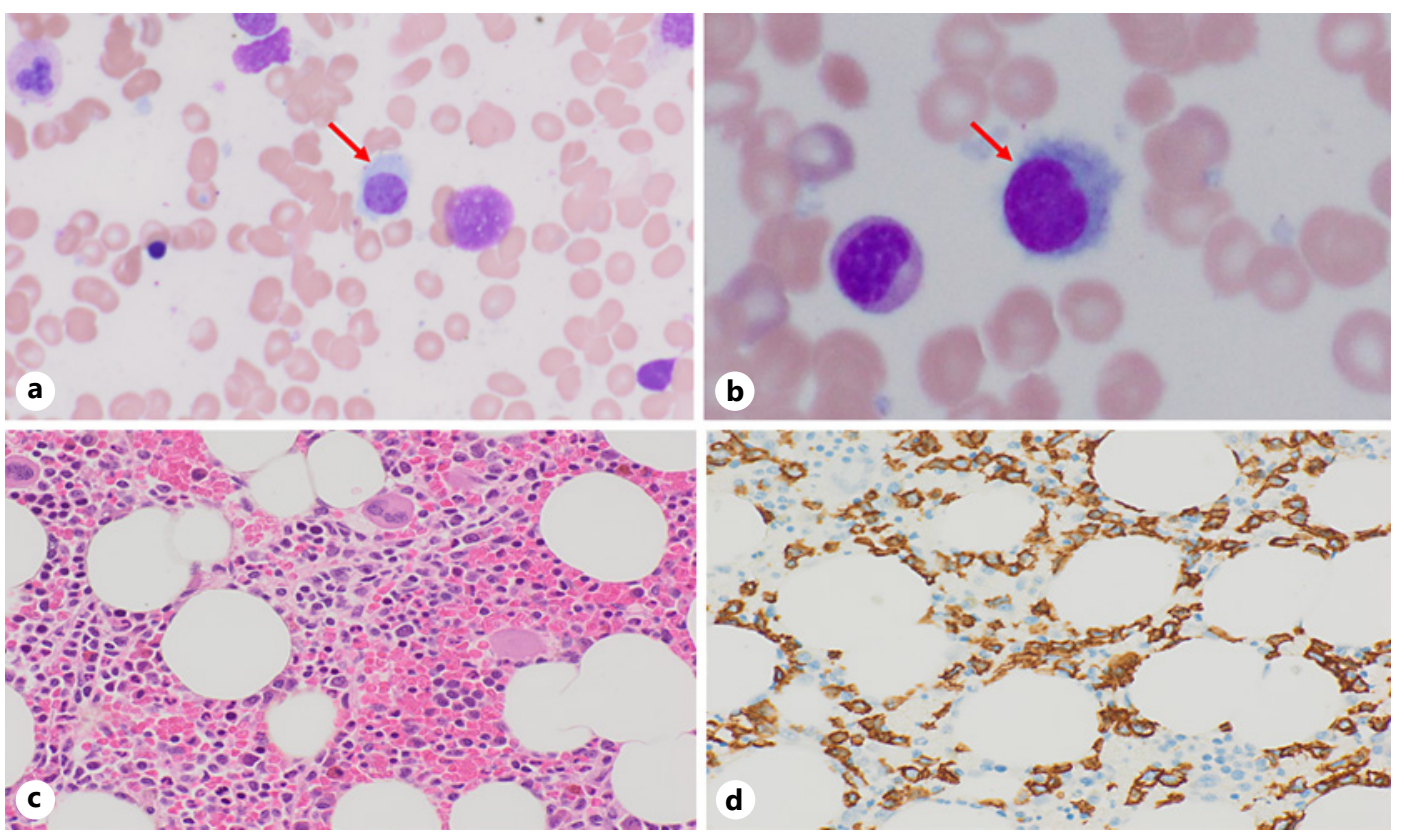

Fig. 3. Immunohistochemistry results of the patient's bone marrow biopsy. Bone marrow aspirate smear showing atypical lymphocytes with hair-like cytoplasmic projections $(\mathbf{a}, \times 40 ; \mathbf{b}, \times 100)$. $\mathbf{c}$ Bone marrow biopsy showing interstitial infiltrate by HCL. d CD20 immunohistochemical highlighting the interstitial leukemic infiltrate in the bone marrow biopsy.

germline or somatic alterations, the olaparib approval includes an expanded list of HRR genes. These alterations can be detected with germline testing and/or CGP testing through analysis of tumor tissue DNA or ctDNA [7].

In this case, liquid-biopsy testing was performed using FoundationOne ${ }^{\circledR}$ Liquid following biochemical and radiographic progression. Tissue CGP testing using FoundationOne ${ }^{\circledR} \mathrm{CDx}$ detects HRR gene alterations from both archived primary tumor specimens and newly acquired metastatic biopsy specimens with high specificity [13]. Liquid biopsy is an effective method of capturing these same alterations, demonstrating high concordance with tissue biopsy [14].

Liquid biopsy testing has the added advantage of capturing genomic changes reflective of acquired treatment resistance and/or clonal selection, as well as the ability to help reveal both intra- and inter-heterogeneity among tumors [7, 10]. Sensitivity and accuracy of liquid biopsy testing are dependent on the extent of ctDNA shedding by the tumor(s), as well as proper sample acquisition after a patient is identified as having progressed through current therapy $[15,16]$.

Results of the FoundationOne ${ }^{\circledR}$ Liquid test for this patient (Table 1) revealed a CHEK2 T367fs*15 (c.1100delC) mutation at a variant allele frequency of 50.85\% that was consistent with the same mutation found through germline testing. The frequency of $C H E K 2$ mutations in prostate cancer is $1.8 \%$ and are included as part of the olaparib approval due to the role of CHEK2 in DNA damage repair via the HRR pathway [4, 6]. Though BRAF alterations overall are found in $\sim 2.2 \%$ of prostate cancer tumors, the BRAF V600E mutation identified in this patient's tumor is exceedingly rare [6]. The BRAF V600E mutation is almost universally present in HCL, and its identification marks a disease-defining genomic event $[17,18]$. In cancer subtypes that are known to have BRAF mutational activity, HCL has by far the highest frequency of V600E events with $>97 \%$ of patients compared to melanomas (60\%), thyroid 
carcinomas (60\%), and a variety of other solid tumors [19]. BRAF plays a role in the RAS-RAFMAPK signaling pathway which regulates cell survival, proliferation, and differentiation. When mutated, $B R A F$ constitutively activates the MEK-ERK pathway and acts as an oncogenic driver through enhanced kinase activity [19]. Kinase inhibitors targeting BRAF V600E mutations, such as vemurafenib, have demonstrated remarkable efficacy with or without rituximab in HCL patients with primary refractory or relapsed/refractory disease after treatment with purine analogs, and this is now considered the standard of care [19-21]. For the patient described in this case report, potential treatment options were discussed during a multidisciplinary tumor board after diagnostic confirmation of HCL. Given this patient was asymptomatic with a stable platelet count (Fig. 2), it was concluded that the more aggressive mCRPC diagnosis should be the focus of treatment while continuing surveillance of HCL. PARPi therapy for mCRPC was initially considered for treatment due to the CHEK2 mutation identified from liquid biopsy. However, treatment with PARPi, in particular olaparib, has been shown to increase the risk of developing delayed myelodysplastic syndrome and acute myeloid leukemia that can often be fatal $[22,23]$. There is little to no data to guide the use of olaparib for mCRPC on patients with underlying chronic lymphoproliferative disorders. Hence, the patient was treated with targeted radiation therapy to the right iliac node, in addition to abiraterone/prednisone, with subsequent decline in PSA.

The use of ctDNA both complements and confers distinct advantages over tissue biopsies. In mCRPC, where disease spread is mostly limited to bone, liquid sampling can capture tumor information without the need for an invasive procedure. Liquid biopsy can also help reveal tumor heterogeneity, both spatially (differences between the primary tumor and metastases) and temporally (differences exposed during disease progression), which are key reasons for treatment failure [10]. In response to the dynamic, selective pressure which arises with treatment, specimens of advanced prostate cancer have been shown to accumulate genomic alterations that differ from the primary tumor [24]. Frequently, these changes include amplification or mutation of the androgen receptor, mutations in DNA repair genes, and loss or mutation of the tumor suppressor genes TP53 and PTEN [16]. ctDNA can be used to identify the genetic and molecular abnormalities present in MCRPC, allow for repeated sampling to monitor therapy response, identify treatment failure, and guide selection of alternative therapeutic agents.

One limitation of ctDNA analysis using liquid biopsy is the possibility of identifying incidental mutations in circulating blood cells, including clonal hematopoiesis (CH). For example, in NSCLC, mutations in JAK2, TP53, and rare mutations in KRAS consistent with CH were detected in the peripheral blood but not tumor [25]. In mCRPC, alterations such as JAK2 and NF1 occur with lower frequency but are also genes frequently associated with $\mathrm{CH}$ when detected in liquid biopsy plasma samples [14]. While CHEK2 alterations have been found to occur more frequently in ctDNA specimens compared to tissue due to both somatic- and CH-related variants [14], genetic testing confirmed the CHEK2 mutation identified in our patient's case was germline. $B R A F$ mutations however are not associated with $\mathrm{CH}$, thereby suggesting a concurrent pathologic hematological process which was ultimately diagnosed by bone marrow biopsy. Future testing should thus incorporate combined peripheral blood DNA and ctDNA analysis to clarify the origin of potentially actionable genomic signals and isolate $\mathrm{CH}$-associated mutations which may confound the therapeutic implications of NGS results.

In conclusion, this case report provides insights into the clinical utility of CGP using liquid biopsy testing to help reveal genomic heterogeneity. The reported $B R A F \mathrm{~V} 600 \mathrm{E}$ mutation is rarely found in prostate cancer patients and suggested the presence of a secondary malignancy later confirmed to be HCL. Though olaparib is approved for mCRPC patients with CHEK2 mutations, the identification of the secondary leukemia diagnosis significantly impacted the therapeutic decision-making for this patient.

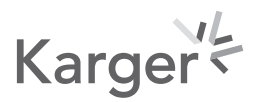




\section{Case Reports in Oncology}

\begin{tabular}{l|l}
\hline Case Rep Oncol 2022;15:78-85 \\
\hline DOI: 10.1159/000521841 & $\begin{array}{l}\text { @ 2022 The Author(s). Published by S. Karger AG, Basel } \\
\text { www.karger.com/cro }\end{array}$ \\
\hline
\end{tabular}

Hemenway et al.: Utility of Liquid Biopsy: A Case Report

\section{Statement of Ethics}

Approval for this study, including a waiver of informed consent and a HIPAA waiver of authorization, was obtained from the Western Institutional Review Board (Protocol No. 20152817). Written informed consent for publication of their clinical details was obtained from the patient.

\section{Conflict of Interest Statement}

Marni B. Tierno is an employee of Foundation Medicine, a wholly owned subsidiary of Roche, and has equity interest in Roche.

\section{Funding Sources}

No funding was received.

\section{Author Contributions}

G.H. annotated the clinical data and wrote the manuscript. M.B.T. reviewed the literature and wrote the manuscript. R.N. cataloged and summarized hematopathology micrographs. R.S. and M.Z. edited the manuscript and supervised the project. All the authors reviewed and approved the final text.

\section{Data Availability Statement}

The authors declare that all data supporting the findings of this study are available within the article. Data sharing is not applicable to this article as no datasets were generated or analyzed during the current study.

\section{References}

1 Abida W, Patnaik A, Campbell D, Shapiro J, Bryce AH, McDermott R, et al. Rucaparib in men with metastatic castration-resistant prostate cancer harboring a BRCA1 or BRCA2 gene alteration. J Clin Oncol. 2020;38(32): 3763-72.

2 de Bono J, Mateo J, Fizazi K, Saad F, Shore N, Sandhu S, et al. Olaparib for metastatic castration-resistant prostate cancer. N Engl J Med. 2020;382(22):2091-102.

3 Hussain M, Mateo J, Fizazi K, Saad F, Shore N, Sandhu S, et al. Survival with olaparib in metastatic castrationresistant prostate cancer. N Engl J Med. 2020;383(24):2345-57.

4 Lynparza (olaparib) [package insert]. Wilmington, DE: AstraZeneca Pharmaceuticals LP; 2020.

5 Rubraca (rucaparib) [package insert]. Boulder, CO: Clovis Oncology, Inc.; 2020.

6 Chung JH, Dewal N, Sokol E, Mathew P, Whitehead R, Millis SZ, et al. Prospective comprehensive genomic profiling of primary and metastatic prostate tumors. JCO Precis Oncol. 2019;3:PO.18.00283.

7 Mateo J, McKay R, Abida W, Aggarwal R, Alumkal J, Alva A, et al. Accelerating precision medicine in metastatic prostate cancer. Nat Cancer. 2020;1(11):1041-53.

8 Corcoran RB, Chabner BA. Application of cell-free DNA analysis to cancer treatment. N Engl J Med. 2018; 379(18):1754-65.

9 Aggarwal C, Rolfo CD, Oxnard GR, Gray JE, Sholl LM, Gandara DR. Strategies for the successful implementation of plasma-based NSCLC genotyping in clinical practice. Nat Rev Clin Oncol. 2021;18(1):56-62.

10 Russano M, Napolitano A, Ribelli G, Iuliani M, Simonetti S, Citarella F, et al. Liquid biopsy and tumor heterogeneity in metastatic solid tumors: the potentiality of blood samples. J Exp Clin Cancer Res. 2020;39(1):95. 
11 Keytruda (pembrolizumab) [package insert]. Whitehouse Station, NJ: Merck \& Co., Inc.; 2020.

12 Marabelle A, Fakih M, Lopez J, Shah M, Shapira-Frommer R, Nakagawa K, et al. Association of tumour mutational burden with outcomes in patients with advanced solid tumours treated with pembrolizumab: prospective biomarker analysis of the multicohort, open-label, phase 2 KEYNOTE-158 study. Lancet Oncol. 2020;21(10):1353-65.

13 Hussain MHA, Mateo J, Sandhu SK, Fizazi K, Saad F, Shore ND, et al. Next-generation sequencing (NGS) of tumor tissue from >4000 men with metastatic castration-resistant prostate cancer (mCRPC): the PROfound phase III study experience. J Clin Oncol. 2020;38(6_Suppl):195.

14 Tukachinsky H, Madison RW, Chung JH, Gjoerup O, Severson EA, Dennis L, et al. Genomic analysis of circulating tumor DNA in 3,334 patients with advanced prostate cancer identifies targetable BRCA alterations and AR resistance mechanisms. Clin Cancer Res. 2021;27(11):3094-105.

15 Conteduca V, Wetterskog D, Scarpi E, Romanel A, Gurioli G, Jayaram A, et al. Plasma tumour DNA as an early indicator of treatment response in metastatic castration-resistant prostate cancer. Br J Cancer. 2020;123(6): 982-7.

16 González-Billalabeitia E, Conteduca V, Wetterskog D, Jayaram A, Attard G. Circulating tumor DNA in advanced prostate cancer: transitioning from discovery to a clinically implemented test. Prostate Cancer Prostatic Dis. 2019;22(2):195-205.

17 Durham BH, Getta B, Dietrich S, Taylor J, Won H, Bogenberger JM, et al. Genomic analysis of hairy cell leukemia identifies novel recurrent genetic alterations. Blood. 2017;130(14):1644-8.

18 Tiacci E, Pettirossi V, Schiavoni G, Falini B. Genomics of hairy cell leukemia. J Clin Oncol. 2017;35(9):1002-10.

19 Zaman A, Wu W, Bivona TG. Targeting oncogenic BRAF: past, present, and future. Cancers. 2019;11(8):1197.

20 National Comprehensive Cancer Network. Hairy cell leukemia (version 2.2021). 2021. Available from: https: //www.nccn.org/professionals/physician_gls/pdf/hairy_cell.pdf.

21 Roider T, Falini B, Dietrich S. Recent advances in understanding and managing hairy cell leukemia. F1000Res. 2018;7:F1000.

22 Master SR, Mansour RP. Myelodysplastic syndrome and acute myeloid leukemia as side effect of PARP inhibitors. J Clin Oncol. 2020;38(15_Suppl):3601.

23 Morice PM, Leary A, Dolladille C, Chrétien B, Poulain L, González-Martín A, et al. Myelodysplastic syndrome and acute myeloid leukaemia in patients treated with PARP inhibitors: a safety meta-analysis of randomised controlled trials and a retrospective study of the WHO pharmacovigilance database. Lancet Haematol. 2021; 8(2):e122-34.

24 Cancer Genome Atlas Research Network. The molecular taxonomy of primary prostate cancer. Cell. 2015; 163(4):1011-25.

25 Hu Y, Ulrich BC, Supplee J, Kuang Y, Lizotte PH, Feeney NB, et al. False-positive plasma genotyping due to clonal hematopoiesis. Clin Cancer Res. 2018;24(18):4437-43. 